\title{
Inhaled heparin in cystic fibrosis
}

\author{
D.J. Serisier*\#", J.K. Shute ", P.M. Hockey", B. Higgins ${ }^{+}$, J. Conway ${ }^{\S}$ and M.P. Carroll ${ }^{\#}$
}

ABSTRACT: Cystic fibrosis (CF) is characterised by inspissated airway secretions and chronic endobronchial infection associated with exuberant neutrophilic inflammation. Unfractionated heparin may be mucolytic and has demonstrated a number of anti-inflammatory properties; however, further safety data are needed in these subjects who are at risk of airway bleeding. The current study aimed to assess the medium-term safety and tolerability of moderately high-dose inhaled heparin in CF adults and to explore possible in vivo mucolytic and anti-inflammatory outcomes.

A randomised, double-blind, placebo-controlled crossover study of twice daily inhalation of 50,000 IU of heparin for 2 weeks was undertaken in CF adults, with a 1-week washout period. Eighteen subjects were randomised and 14 (mean \pm SD age $23 \pm 7.8$ yrs and percentage-predicted forced expiratory volume in one second $52.1 \pm 15.56 \%$ ) completed the study protocol.

Heparin neither affected blood coagulation parameters nor resulted in any increase in adverse events. Heparin inhalation had no significant effect upon forced expiratory volume in one second, symptoms of sputum clearance or sputum inflammatory markers.

The current pilot study demonstrated no evidence of improved sputum clearance with 50,000 IU of inhaled heparin given twice daily to adult cystic fibrosis subjects. However, inhaled heparin was safe and the future evaluation of larger doses over a longer period may be warranted.

KEYWORDS: Cystic fibrosis, heparin, inflammation, mucolytic, neutrophil elastase

I

nspissated respiratory secretions and chronic airway inflammation are central to the progressive pulmonary damage that characterises cystic fibrosis (CF), ultimately culminating in respiratory failure and death. Intense neutrophilic activity is central to the inflammatory process [1], augmented by excessive levels of proinflammatory cytokines [2], particularly interleukin (IL)-8 [3], and the destructive inflammatory serine protease, neutrophil elastase [4].

The systemic anti-inflammatory therapies prednisone and ibuprofen both improve lung function in $\mathrm{CF}[5,6]$; however, neither appears to be effective in adults [5, 7] and both have significant potential side-effects. Macrolide antibiotics have shown interesting anti-inflammatory properties, although the mechanism of their clinical efficacy in CF subjects remains unclear [8].

Enhanced clearance of retained secretions using recombinant human deoxyribonuclease (DNase) also improves lung function in CF [9]. Furthermore, improved clearance of respiratory secretions may reduce airway inflammation, as DNase appears to attenuate the progressive increase in airway inflammation that occurs in CF [10]. However, DNase is expensive, and a substantial proportion of CF subjects do not appear to derive benefit [11].
There are reports of increased sputum expectoration with unfractionated heparin [12, 13], and charged oligosaccharides have shown mucolytic effects in vitro [14], suggesting that heparin may enhance airway clearance. Furthermore, heparin has displayed a diverse range of in vitro antiinflammatory and immunomodulatory effects, including inhibitory effects upon leukocyte recruitment [15], neutrophil chemotaxis [16], neutrophil elastase [17] and chemokines, such as IL-8 [18]. Excess neutrophil elastase activity plays a key role in contributing to airway obstruction in CF by multiple mechanisms, including stimulating IL-8 production [19] and mucus secretion [20], impairing ciliary function [21], and potentially desiccating airway secretions by activating epithelial sodium channels [22]. Recently, the current authors observed significant reductions in sputum neutrophil elastase activity ex vivo with increasing doses of inhaled heparin in a small group of CF subjects [23], with several subjects commenting upon immediate enhancement of sputum clearance following the higher doses.

Despite the recent demonstration of the safety of single high doses of inhaled heparin in normal subjects [24], CF subjects have specific safety issues related to airway bleeding. The current study aimed to assess the medium-term safety

\section{AFFILIATIONS}

*Dept of Respiratory Medicine, Mater Adult Hospital, Brisbane, Australia.

"Adult Cystic Fibrosis Unit,

Southampton General Hospitals NHS Trust, and

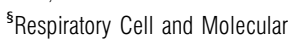
Biology Division, Southampton University, Southampton,

"School of Pharmacy and Biomedical Sciences, and

+Dept of Mathematics, University of Portsmouth, Portsmouth, UK.

\section{CORRESPONDENCE}

D.J. Serisier

Dept of Respiratory Medicine

Mater Adult Hospital

Brisbane, 4101

Australia

Fax: 61738402402

E-mail: david_serisier@mater.org.au

Received:

June 132005

Accepted after revision:

October 032005

European Respiratory Journal

Print ISSN 0903-1936

Online ISSN 1399-3003 
and tolerability of moderately high dose inhaled unfractionated heparin in CF adults and to explore possible in vivo mucolytic (assessed by lung function, symptom scores and quality of life) and anti-inflammatory outcomes. The study was powered to detect a 10\% improvement in forced expiratory volume in one second (FEV1) as a result of enhanced sputum clearance, consistent with the short-term effect demonstrated in early studies of DNase [25].

\section{METHODS}

\section{Subjects}

Nonsmoking adults with CF were recruited from the adult CF clinic at Southampton General Hospital, Southampton, UK, if they had been clinically stable for 21 days. The protocol was approved by the hospital ethics committee and all subjects provided written informed consent. At enrolment, FEV1 was required to be within $10 \%$ of the best recorded value in the preceding 6 months.

Exclusion criteria were pregnancy, allergy to heparins, or any of the following within the preceding 21 days: symptoms of infective exacerbation; prescription of supplemental antibiotic therapy; and change in maintenance therapies or haemoptysis. Subjects with a bleeding diathesis, including platelet count $<100 \times 10^{6}$ cells $\cdot \mathrm{mL}^{-1}$, or international normalised ratio or activated partial thromboplastin ratio $>1.25$, were also excluded.

\section{Study design and procedures}

This was a randomised, double-blind, placebo-controlled crossover study. Subjects were allocated in random order to consecutive 2-week treatments of inhaled, twice daily, 4-mL $0.9 \%$ saline or 50,000 IU of heparin sodium in injectable form $\left(25,000 \mathrm{IU} \cdot \mathrm{mL}^{-1}\right.$; Leo Laboratories, Princes Risborough, UK; made up to $4 \mathrm{~mL}$ with $0.9 \%$ sodium chloride solution). All study treatments were administered with a Sidestream jet nebuliser and Portaneb compressor (Medic-Aid, Bognor Regis, $\mathrm{UK})$, after taking short-acting $\beta$-agonist and performing usual chest physiotherapy. All usual medications were maintained throughout the entire trial period. Subjects were directed to return unused doses at the completion of each of the treatments to enable an estimation of treatment compliance.

Randomisation was performed using computer-generated block randomisation in groups of four, supplied by the Statistics Dept and held by the Pharmacy Dept. The Pharmacy Dept allocated, prepared and supplied trial drug to sequential subjects according to their randomisation code. Trial subjects, trial supervisors and all staff involved in direct patient care were unaware of treatment allocations at all times. Code-breaking and allocation sheets for emergency use were kept by the Pharmacy Dept, but were never used. Heparin and saline were provided as identical, clear, colourless solutions.

The dose of heparin sodium selected was based upon the current authors' preceding pilot study [23], which showed significant reductions in sputum neutrophil elastase activity following single-inhaled doses of both 1,000 and 2,000 IU $\cdot \mathrm{kg}^{-1}$. There was a 1-week washout period between treatments. In the event that any subject's FEV1 at the start of the second treatment was $<90 \%$ of their baseline FEV1 at commencement of treatment one, the washout period was prolonged to allow administration of 14 days of antibiotic therapy, followed by a further 21-day period of clinical stability prior to commencing treatment two. This maximised the equivalence of subjects for the two treatments.

Withdrawal criteria included haemoptysis $\geqslant 20 \mathrm{~mL}$, pulmonary infective exacerbation or any requirement for antibiotic therapy during treatment periods. However, heparin inhalation has been associated with increased sputum expectoration $[12,13]$, which may result in subjects interpreting a decline in their respiratory status. In an attempt to minimise the possible influence of such a subjective effect upon trial outcomes (and given that there is no universally agreed definition for an infective exacerbation), infective exacerbation was defined as a requirement for oral or intravenous antibiotic therapy associated with a $10 \%$ or greater decline in FEV1 and increase in serum C-reactive protein (CRP; of at least $10 \mathrm{mg} \cdot \mathrm{L}^{-1}$ and at least $20 \%$ from baseline), in addition to at least two of the following: increased sputum purulence; increased sputum volume; new or increased haemoptysis; fevers or systemic symptoms; weight loss of at least $1 \mathrm{~kg}$; new crackles on chest auscultation; and new infiltrates on chest radiograph.

Safety was assessed by both blood coagulation indices and incidence of adverse events. The primary outcome measure was change in FEV1, comparing heparin and placebo treatments. Secondary outcome measures were changes in the following: sputum levels of neutrophil elastase activity, IL-8, terminal complement complex (TCC) and myeloperoxidase (MPO); serum CRP; weight; symptom scores; quality of life and exercise capacity.

Spirometry was performed according to American Thoracic Society criteria [26], using a Sensormedics rolling seal spirometer (Sensormedics; Viasys Healthcare, Yorba Linda, CA, USA). Timing of spirometry was kept consistent to within $2 \mathrm{~h}$ during the trial. To assess exercise tolerance, patients underwent a maximal, incremental $10-\mathrm{m}$ shuttle walking test, as previously described [27]. Visual analogue scores for eight symptoms (general well-being, energy, appetite, sleep quality, shortness of breath, cough, sputum volume and sputum colour) were completed at each visit without reference to prior scores or answers. Subjects also completed the teenadult version of the Cystic Fibrosis Questionnaire (CFQ), a validated, disease-specific, health-related quality-of-life measure [28].

At each study visit, spontaneously expectorated sputum samples were collected in airtight sterile containers and stored at $-80^{\circ} \mathrm{C}$ for subsequent processing. After thawing samples on ice, salivary contamination was removed by pipette and the sample diluted $\left(1: 1 \mathrm{~mL} \cdot \mathrm{g}^{-1}\right)$ with PBS (Gibco, Paisley, UK) for solubilisation of mediators. The samples were thoroughly vortexed on a whirly mixer for $5 \mathrm{~min}$, mixed on an orbital plate mixer for $20 \mathrm{~min}$ on ice, then centrifuged at $20,000 \times g$, at $4{ }^{\circ} \mathrm{C}$ for $20 \mathrm{~min}$. The supernatant was aspirated and used to determine neutrophil elastase activity, IL-8, MPO and TCC levels. Commercially available ELISA kits were used to measure total IL-8 (Pelikine compact; Mast Diagnostics, Liverpool, UK) and TCC (Quidel, San Diego, CA, USA). MPO was measured using a commercially available RIA kit (Pharmacia, Uppsala, Sweden). Neutrophil elastase activity 
was measured in samples diluted 1:10 with assay buffer, as previously described [29].

\section{Statistical analysis}

The current authors' sample size calculation indicated the need for a minimum of 13 evaluable patients to detect a difference in change in FEV1 of $0.14 \mathrm{~L}$ (equivalent to $10 \%$ difference in mean scores), with $95 \%$ power at the $5 \%$ (two-sided) level of significance. This assumed a SD of $0.123 \mathrm{~L}$ for the difference between paired observations estimated from the current authors' pilot data [22].

The sample size was increased to 18 subjects (a 30\% increase) to allow for attrition. To be eligible for full outcome analysis, a patient had to be recruited, randomised, and had to complete both arms of the trial without dropout; however, safety data were collected for all subjects. All measurements, data collection and data entry were completed before treatment codes were broken.

Analyses compared within-subject differences for changes in outcomes between heparin and placebo. For all such differences in change that were normally distributed, a complete crossover ANOVA for repeated measures was performed, including assessment for period effects and treatment-period interaction. If there was evidence of period effect or treatmentperiod interaction, covariate analyses were performed upon treatment period one data alone, treating the data as per a parallel study. The Wilcoxon signed-rank test was employed for non-normally distributed outcome data. Comparison of groups for determination of differences in the incidence of side-effects and baseline variables used McNemar's test for analysis of paired proportions. Pearson's product moment correlation coefficients were computed to assess the degree of association between numeric variables on appropriate measurement scales. p-Values $<0.05$ were considered significant.

\section{RESULTS}

Eighteen subjects were randomised and four were subsequently withdrawn, two in relation to heparin treatment (both infective exacerbation) and two in relation to placebo (one for noncompliance and one for personal reasons). The 14 remaining subjects completed both treatment periods; eight received heparin first and six received placebo first. Table 1 shows the patient characteristics. There were no differences in the clinical characteristics of the subjects at the start of the heparin and placebo periods. Four subjects had their washout periods prolonged; three received oral antibiotics and one received intravenous antibiotics.

The average compliance was $95 \%$ for heparin and $96 \%$ for placebo. There were no significant adverse events attributable to heparin. The incidence of side-effects did not differ between heparin and placebo. No subject experienced haemoptysis whilst inhaling heparin. No subject discontinued the trial due to treatment intolerance on either arm. Heparin had no effect upon blood coagulation indices, including platelet count (treatment effect $-7.1 \times 10^{6}$ cells $\cdot \mathrm{mL}^{-1} ; \mathrm{p}=0.67 ; 95 \%$ confidence interval (CI) -42.7-28.5), activated partial thromboplastin time or international normalised ratio. Infective exacerbations developed in eight of the 18 randomised subjects during the study, three during treatment and five during washout. Five
TABLE 1 Baseline characteristics of the study population

\begin{tabular}{|c|c|}
\hline & Average \\
\hline Age yrs & $23.8 \pm 7.8$ \\
\hline Female $n$ & 10 \\
\hline FEV 1 L & $1.71 \pm 0.52$ \\
\hline FEV $1 \%$ & $52.7 \pm 16$ \\
\hline Weight kg & $55.3 \pm 8.4$ \\
\hline Body mass index $\mathrm{kg} \cdot \mathrm{m}^{-2}$ & $20.5 \pm 2$ \\
\hline \multicolumn{2}{|l|}{ Microbiology $\mathbf{n}^{\#}$} \\
\hline Pseudomonas aeruginosa & 11 \\
\hline Burkholderia cepacia & 1 \\
\hline Staphylococcus aureus & 3 \\
\hline \multicolumn{2}{|l|}{ Medications $\mathbf{n}$} \\
\hline Bronchodilators & 9 \\
\hline Inhaled corticosteroids & 11 \\
\hline Inhaled antibiotics & 12 \\
\hline Inhaled deoxyribonuclease & 1 \\
\hline Azithromycin & 3 \\
\hline
\end{tabular}

Data are presented as mean $\pm \mathrm{SD}$, unless otherwise indicated. FEV1: forced expiratory volume in one second; FEV $1 \%$ : FEV 1 as a percentage of the predicted value. ${ }^{\#}$ : subjects with chronic pulmonary colonisation by the indicated organism (some subjects were colonised with $>1$ organism). $n=14$.

occurred in relation to heparin treatment and three in relation to placebo $(\mathrm{p}=0.73)$.

There were no significant differences between the heparin and placebo arms for incidence of cough $(p=0.50)$ or unusual taste $(p=0.63)$ with inhalation of trial drug. Visual analogue scores for cough did not differ significantly between heparin and placebo (treatment effect $-0.08 ; \mathrm{p}=0.87 ; 95 \% \mathrm{CI}-1.11-0.95$ ).

\section{Outcomes}

There was no significant effect of heparin upon the primary outcome measure, FEV1 (treatment effect $-0.008 \mathrm{~L} ; \mathrm{p}=0.90 ; 95 \%$ CI $-0.15-0.13$; table 2 ), or the forced vital capacity or inspiratory capacity. There was a trend to treatment by period interaction for FEV1 $(p=0.061)$; however, covariate analysis performed on treatment period one did not alter any results (mean difference $-0.08 \mathrm{~L} ; \mathrm{p}=0.36$; 95\% CI $-0.26-0.1$; table 2). Heparin inhalation was associated with individual improvements in FEV1 of $\geqslant 10 \%$ in two subjects and a decline of $\geqslant 10 \%$ in one.

There was no significant effect of heparin upon visual analogue scores (including those pertaining to sputum clearance; table 2), overall quality of life on the CFQ, or any of the three quality-of-life modules. Heparin was associated with negative effects upon both the body image (median difference $-5.56 ; p=0.04)$ and social/marginalisation dimensions (median difference $-6.67 ; \mathrm{p}=0.07$ ); however, these were not significant after incorporating a multiple comparisons adjustment for the 15 quality-of-life measures of the CFQ (after a Bonferroni adjustment, significance at the $5 \%$ level is indicated by a p-value $<0.003$ ).

There were no significant effects of heparin inhalation upon weight, serum CRP or exercise tolerance. Inhaled heparin had no significant effect upon any of the sputum inflammatory markers (table 2). 
TABLE 2 Changes in selected outcome measures according to treatment regimen

\begin{tabular}{|c|c|c|c|c|}
\hline & Heparin & Placebo & Treatment effect ${ }^{\#}$ & p-value \\
\hline \multicolumn{5}{|l|}{ Lung function ${ }^{f}$} \\
\hline FEV 1 L & $-0.03(-0.13-0.07)$ & $0.05(-0.04-0.14)$ & $-0.08(-0.26-0.1)$ & $0.36^{+}$ \\
\hline \multicolumn{5}{|l|}{ Sputum symptom scores } \\
\hline Sputum volume & $0.28(-0.78-1.34)$ & $-0.01(-0.76-0.74)$ & $0.22(-1.26-1.71)$ & $0.75^{\circ}$ \\
\hline $\mathrm{NE}$ activity $\mathrm{mU} \cdot \mu \mathrm{L}^{-1}$ & $1.3(-8.9-11.5)$ & $-3.3(-8.7-2.1)$ & $5.55(-7.3-18.3)$ & $0.36^{\circ}$ \\
\hline Total IL-8 $\times 10^{2} \mathrm{ng} \cdot \mathrm{mL}^{-1}$ & $0.19(-27.3-48.9)$ & $-0.12(-4.9-8.4)$ & $0.30(-35.7-53.8)$ & $0.70^{\S}$ \\
\hline MPO $\mathrm{mg} \cdot \mathrm{L}^{-1}$ & $15.9(-29.86-61.66)$ & $-12.5(-44-19)$ & $32.72(-35.2-100.7)$ & $0.31^{\#}$ \\
\hline TCC $n g \cdot \mathrm{mL}^{-1}$ & $16.85(-55.45-89.15)$ & $-19.4(-59.7-20.9)$ & $-2.85(-38.8-33.1)$ & $0.87^{\#}$ \\
\hline
\end{tabular}

\section{DISCUSSION}

The current study investigates both the largest dose and longest duration of inhaled heparin yet reported in any clinical trial of subjects with lung disease. Despite evaluating a group of patients potentially at risk of pulmonary bleeding, inhaled heparin was safe and well tolerated, with no bleeding complications. Furthermore, consistent with the short half-life of heparin and data demonstrating little systemic absorption from the tracheobronchial tree, there was no evidence of any cumulative effect of heparin upon systemic coagulation markers, extending previous findings [12, 24, 30, 31].

This trial failed to detect any clinical benefit of 2 weeks of heparin inhalation in chronically productive adult CF subjects with moderate lung disease. The study was powered to detect a large treatment effect of inhaled heparin on FEV1 and the treatment duration was necessarily limited for safety reasons. Therefore, this study was not designed or powered adequately to exclude an effect of heparin upon clinical measures, including lung function. Although no evidence of enhanced sputum clearance (on the basis of symptom scores, quality of life or lung function) was found, recent work within the current authors' laboratory provides further evidence of mucolytic activity of unfractionated heparin in vitro (unpublished data), and a longer treatment duration or larger dose may therefore be needed to result in mucolysis in vivo.

The inability to demonstrate any ex vivo anti-inflammatory effect of heparin contrasts with prior demonstrations of rapid reductions in neutrophil elastase activity [23] and IL-8 and IL-6 [12] in sputum. It is possible that this is the result of insufficient dosing or inadequate duration, although reductions in sputum inflammatory markers were previously shown with only 25,000 IU of inhaled heparin given once daily for 7 days [12] and with single doses of both of the higher doses $(1,000$ and $2,000 \mathrm{IU} \cdot \mathrm{kg}^{-1}$ ) in the current authors' pilot study [23]. The substantial variability in measures of inflammatory mediators in spontaneously expectorated sputum from CF subjects [32] may have resulted in spurious findings in those prior uncontrolled studies, and further dose-ranging studies are needed.

In conclusion, inhaled heparin at a dose of 50,000 IU administered twice daily for 2 weeks was well tolerated in cystic fibrosis adults and did not result in any bleeding complications or evidence of adverse events. This provides support for its future cautious evaluation in larger doses, for longer periods, or in subjects with early lung disease.

\section{REFERENCES}

1 Muhlebach MS, Stewart PW, Leigh MW, Noah TL. Quantification of inflammatory responses to bacteria in young cystic fibrosis and control patients. Am J Respir Crit Care Med 1999; 160: 186-191.

2 Bonfield TR, Panuska JR, Konstan MW, et al. Inflammatory cytokines in cystic fibrosis lungs. Am J Respir Crit Care Med 1995; 152: 2111-2118.

3 Dean TP, Dai Y, Shute JK, Church MK, Warner JO. Interleukin-8 concentrations are elevated in bronchoalveolar lavage, sputum, and sera of children with cystic fibrosis. Pediatr Res 1993; 34: 159-161.

4 Meyer KC, Lewandoski JR, Zimmerman JJ, Nunley D, Calhoun WJ, Dopico GA. Human neutrophil elastase and elastase/alpha1-antiprotease complex in cystic fibrosis. Am Rev Respir Dis 1991; 144: 580-585.

5 Konstan MW, Byard PJ, Hoppel CL, Davis PB. Effect of high-dose ibuprofen in patients with cystic fibrosis. $N$ Engl J Med 1995; 332: 848-854.

6 Eigen H, Rosenstein BJ, FitzSimmons S, Schidlow DV. A multicenter study of alternate day prednisone therapy in patients with cystic fibrosis. J Pediatr 1995; 126: 515-523.

7 Pantin CF, Stead RJ, Hodson ME, Batten JC. Prednisolone in the treatment of airflow obstruction in adults with cystic fibrosis. Thorax 1986; 41: 34-38. 
8 Wolter J, Seeney S, Bell S, Bowler S, Masel P, McCormack J. Effect of long term treatment with azithromycin on disease parameters in cystic fibrosis: a randomised trial. Thorax 2002; 57: 212-216.

9 Fuchs HJ, Borowitz DS, Christiansen DH, et al. Effect of aerosolized recombinant human DNase on exacerbations of respiratory symptoms and on pulmonary function in patients with cystic fibrosis. N Engl J Med 1994; 331: 637-642.

10 Paul K, Rietschel E, Ballmann M, et al. Bronchoalveolar lavage for the evaluation of anti-inflammatory treatment study group. Effect of treatment with dornase alpha on airway inflammation in patients with cystic fibrosis. Am J Respir Crit Care Med 2004; 169: 719-725.

11 Davies J, Trindade MT, Wallis C, Rosenthal M, Crawford O, Bush A. Retrospective review of the effects of rhDNase in children with cystic fibrosis. Pediatr Pulmonol 1997; 23: 243-248.

12 Ledson M, Gallagher M, Hart CA, Walshaw M. Nebulised heparin in Burkholderia cepacia colonised adult cystic fibrosis patients. Eur Respir J 2001; 17: 36-38.

13 Youngchaiyud P, Kettel L, Cugell DW. The effect of heparin aerosols on airway conductance in patients with chronic obstructive pulmonary disease. Am Rev Respir Dis 1969; 99: 449-452.

14 King M, Rubin BK. Pharmacologic approaches to discovery and development of new mucolytic agents. Adv Drug Deliv Rev 2002; 54: 1475-1490.

15 Tyrrell DJ, Horne AP, Holme KR, Preuss JMH, Page CP. Heparin in inflammation: potential therapeutic applications beyond anticoagulation. Adv Pharmacol 1999; 46: 151-208.

16 Matzner Y, Marx G, Drexler R, Eldor A. The inhibitory effect of heparin and related glycosaminoglycans on neutrophil chemotaxis. Thromb Haemost 1984; 52: 134-137.

17 Volpi N. Inhibition of human leukocyte elastase activity by heparins: influence of charge density. Biochim Biophys Acta 1996; 1290: 299-307.

18 Ramdin L, Perks B, Sheron N, Shute JK. Regulation of interleukin- 8 binding and function by heparin and alpha2macroglobulin. Clin Exp Allergy 1998; 28: 616-624.

19 Nakamura H, Yoshimura K, McElvaney NG, Crystal RG. Neutrophil elastase in respiratory epithelial lining fluid of individuals with cystic fibrosis induces interleukin- 8 gene expression in a human bronchial epithelial cell line. J Clin Invest 1992; 84: 1478-1484.
20 Schuster A, Ueki I, Nadel JA. Neutrophil elastase stimulates tracheal submucosal gland secretion that is inhibited by ICI 200,355. Am J Physiol 1992; 262: L86-L91.

21 Amitani R, Wilson R, Rutman A, et al. Effects of human neutrophil elastase and Pseudomonas aeruginosa proteinases on human respiratory epithelium. Am J Respir Cell Mol Biol 1991; 4: 26-32.

22 Caldwell RA, Boucher RC, Stutts MJ. Neutrophil elastase activates near-silent epithelial $\mathrm{Na}^{+}$-channels and increases airway epithelial $\mathrm{Na}^{+}$-transport. Am J Physiol Lung Cell Mol Physiol 2005; 288: L813-L819.

23 Shute JK, Forsyth C, Hockey PM, Carroll MP. Antiinflammatory effects of nebulised heparin in cystic fibrosis [abstract]. Am J Respir Crit Care Med 2000; 161: A75.

24 Bendstrup KE, Gram J, Jensen JI. Effect of inhaled heparin on lung function and coagulation in healthy volunteers. Eur Respir J 2002; 19: 606-610.

25 Ramsey BW, Astley SJ, Aitken ML, et al. Efficacy and safety of short-term administration of aerosolised recombinant human deoxyribonuclease in patients with cystic fibrosis. Am Rev Respir Dis 1993; 148: 145-151.

26 American Thoracic Society. Standardization of spirometry - 1994 update. Am J Respir Crit Care Med 1995; 152: 1107-1136.

27 Singh SJ, Morgan MD, Hardman AE, Rowe C, Bardsley PA. Comparison of oxygen uptake during a conventional treadmill test and the shuttle walking test in chronic airflow limitation. Eur Respir J 1994; 7: 2016-2020.

28 Quittner AL, Sweeny S, Watrous M, et al. Translation and linguistic validation of a disease-specific quality of life measure for cystic fibrosis. J Pediatr Psychol 2000; 25: 403-414.

29 Suri R, Marshall LJ, Wallis C, Metcalfe C, Bush A, Shute JK. Effects of recombinant human DNase and hypertonic saline on airway inflammation in children with cystic fibrosis. Am J Respir Crit Care Med 2002; 166: 352-355.

30 Bowler SD, Smith SM, Lavercombe PS. Heparin inhibits the immediate response to antigen in the skin and lungs of allergic subjects. Am Rev Respir Dis 1993; 147: 160-163.

31 Ahmed T, Garrigo J, Danta I. Preventing bronchoconstriction in exercise-induced asthma with inhaled heparin. $N$ Engl J Med 1993; 329: 90-95.

32 Salva PS, Doyle NA, Graham L, Eigen H, Doerschuck CM. TNF-alpha, IL-8, soluble ICAM-1, and neutrophils in sputum of cystic fibrosis patients. Pediatr Pulmonol 1996; 21: 11-19. 